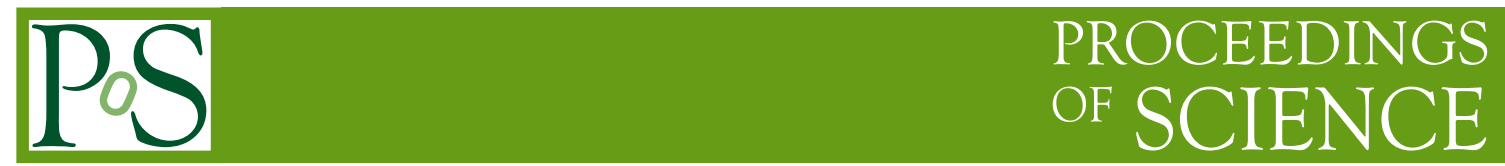

\title{
An improved action for heavy quarks
}

\author{
Andreas S. Kronfeld* \\ Theoretical Physics Department, Fermi National Accelerator Laboratory, Batavia, Illinois, USA \\ E-mail: ask AT fnal.gov
}

\section{Mehmet B. Oktay}

School of Mathematics, Trinity College, Dublin, Ireland

E-mail: oktay AT maths.tcd.ie

We extend the Fermilab method for heavy quarks to include all interactions of dimension six in the action. We discuss a subtlety in the power counting, which implies that, for heavy quarks, certain interactions of dimension seven are commensurate with some of those of dimension six. We then present tree-level matching conditions obtained from calculating the Compton scattering amplitude for (lattice) QCD. When the matching conditions have been applied, the improved action removes (tree-level) discretization errors of order $a^{2} \boldsymbol{p}^{3} / m_{Q}$ and $a^{3} \boldsymbol{p}^{3}$.

XXIVth International Symposium on Lattice Field Theory

July 23-28, 2006

Tucson, Arizona, USA

* Speaker. 


\section{Introduction}

Discretization errors are an important source of uncertainty in lattice QCD calculations with heavy quarks. As with gluons and light quarks, one uses effective field theory to understand and control discretization (aka cutoff or lattice-spacing) effects. With heavy quarks there are two possibilities: a (modified) Symanzik effective Lagrangian and a (modified) heavy-quark effective Lagrangian (HQET for heavy-light hadrons; NRQCD for quarkonium).

Usually, the Symanzik local effective Lagrangian (LEL $\mathscr{L}$ ) is devised with only one short distance in mind, the lattice spacing $a$. Similarly, HQET/NRQCD is usually constructed with only the heavy quark's (or quarks') Compton wavelength(s), $1 / m_{Q}$, as short distance(s). With heavy quarks on a lattice one must consider both kinds of short distances. In particular, couplings in the lattice Lagrangian and Wilson coefficients in the effective Lagrangians depend on the dimensionless ratio(s) $m_{Q} a$.

Treating the inverse quark mass as a short distance, the Symanzik LE $\mathscr{L}$ can be written [1]

$$
\begin{aligned}
\mathscr{L}_{\text {Sym }} & =-\bar{q}\left(\gamma_{4} D_{4}+\sqrt{\frac{m_{1}}{m_{2}}} \boldsymbol{\gamma} \cdot \boldsymbol{D}+m_{1}\right) q+a K_{t}^{\text {lat }} \bar{q}\left(\gamma_{4} D_{4}+m_{1}\right)^{2} q+a K_{s}^{\text {lat }} \bar{q} \boldsymbol{D}^{2} q \\
& +a K_{B}^{\text {lat }} \bar{q} i \boldsymbol{\Sigma} \cdot \boldsymbol{B} q+a K_{E}^{\mathrm{lat}} \bar{q} \boldsymbol{\alpha} \cdot \boldsymbol{E} q+\cdots,
\end{aligned}
$$

where $m_{1}$ is the renormalized rest mass, and mass-dependent short-distance effects are lumped into $\sqrt{m_{1} / m_{2}}$ and the coefficients $K^{\text {lat }}$. An improved action is devised by adjusting $\mathscr{L}_{\text {Sym }}$ to reproduce QCD. Some coefficients may be made to vanish via field redefinitions, such as $K_{t}^{\text {lat }}$ and $K_{s}^{\text {lat }}[2,3]$. The others must be addressed by matching the underlying lattice Lagrangian:

$$
m_{1}=m_{2}=m_{Q}, \quad K_{B}^{\text {lat }}\left(c_{B}\right)=0, \quad K_{E}^{\text {lat }}\left(c_{E}\right)=0, \ldots
$$

where $m_{Q}$ is the physical quark mass, and $c_{B}$ and $c_{E}$ are couplings of dimension-five interactions in the Fermilab lattice action for heavy quarks [3].

Treating the lattice spacing and inverse heavy quark mass as short distances, the heavy-quark effective Lagrangian is [4]

$$
\begin{aligned}
\mathscr{L}_{\mathrm{HQ}} & =-\bar{h}^{( \pm)}\left(\gamma_{4} D_{4}+m_{1}\right) h^{( \pm)}+\frac{\bar{h}^{( \pm)} \boldsymbol{D}^{2} h^{( \pm)}}{2 m_{2}}+\frac{Z_{B}^{\mathrm{lat}} \bar{h}^{( \pm)} i \boldsymbol{\Sigma} \cdot \boldsymbol{B} h^{( \pm)}}{2 m_{2}} \\
& +\frac{Z_{E}^{\mathrm{lat}} \bar{h}^{( \pm)} i \boldsymbol{\Sigma} \cdot[\boldsymbol{D} \times \boldsymbol{E}] h^{( \pm)}}{8 m_{2}^{2}}+\frac{Z_{D}^{\mathrm{lat}} \bar{h}^{( \pm)} \boldsymbol{D} \cdot \boldsymbol{E} h^{( \pm)}}{8 m_{2}^{2}}+\cdots,
\end{aligned}
$$

where the $Z^{\text {lat }}$ capture the short-distance effects. In parallel, one can construct this effective Lagrangian for continuum QCD, with the same operators but different coefficients $Z^{\text {cont }}$. Now improvement is achieved by choosing improvement couplings in the underlying lattice Lagrangian, so that the two effective HQ Lagrangians coincide. That is

$$
m_{2}=m_{Q}, \quad Z_{B}^{\text {lat }}\left(c_{B}\right)=Z_{B}^{\text {cont }}, \quad Z_{E}^{\text {lat }}\left(c_{E}\right)=Z_{E}^{\text {cont }}, \ldots
$$

Both sets of conditions, Eqs. (1.2) or Eqs. (1.4), yield the same results for the improvement couplings $c_{B}$ and $c_{E}$. 
In this paper we focus on an extension of the Fermilab method to include all interactions of dimension six. Some aspects have been reported earlier [5, 6]. Here, we first discuss a subtlety in the power counting, which implies that we must also consider certain interactions of dimension seven to be commensurate with some of those of dimension six.

\section{Power Counting}

With heavy quarks one must pay special attention to power counting. This is simplest in heavy-light hadrons, where the typical three-momentum in the rest frame is $p \approx \Lambda_{\mathrm{QCD}}$. Then it is useful to define $n_{\Gamma}$, which is 0 or 1 , depending on whether the Dirac matrix $\Gamma$ in a quark bilinear commutes or anticommutes with $\gamma_{4}$. A bilinear of dimension $d$ introduces physical effects of order

$$
\left(a \Lambda_{\mathrm{QCD}}\right)^{d-4}\left(\frac{\Lambda_{\mathrm{QCD}}}{m_{Q}}\right)^{n_{\Gamma}} \sim\left(a \Lambda_{\mathrm{QCD}}\right)^{d-4+n_{\Gamma}} \sim\left(\frac{\Lambda_{\mathrm{QCD}}}{m_{Q}}\right)^{d-4+n_{\Gamma}},
$$

which are all the same once one allows for coefficient functions that depend on $m_{Q} a$, with $m_{Q} a$ of order unity. This power counting has recently been considered by Christ, Li, and Lin [7]. It is useful for hadrons with one heavy quark, but for quarkonium one should adopt the power counting of nonrelativistic QCD [8].

We shall denote terms in the lattice action by $S_{\left(d, n_{\Gamma}\right)}$ to classify them by their power counting (2.1). The terms with $n_{\Gamma}=1$ are necessary to ensure a smooth limit when $m_{Q} a \rightarrow 0$ [3], which is a feature distinguishing the Fermilab method from lattice NRQCD. Ref. [3] treated $S_{(5,0)}$ and $S_{(5,1)}$ (and cursorily $S_{(6,0)}$ ). Here we complete the analysis presented at earlier Lattice symposia [5,6] to encompass the full set of interactions in $S_{(6,1)}$ and $S_{(7,0)}$.

\section{Improved Fermilab Action}

The Fermilab action is a generalization of the Sheikholeslami-Wohlert action. We write

$$
S=S_{0}+\sum_{d=5}^{\infty} \sum_{n_{\Gamma}=0}^{1} S_{\left(d, n_{\Gamma}\right)}
$$

where

$$
\begin{aligned}
S_{0}= & m_{0} a^{4} \sum_{x} \bar{\psi}(x) \psi(x)+a^{4} \sum_{x} \bar{\psi}(x) \gamma_{4} D_{4 \text { lat }} \psi(x)-\frac{1}{2} a^{5} \sum_{x} \bar{\psi}(x) \triangle_{4 \text { lat }} \psi(x) \\
& +\zeta a^{4} \sum_{x} \bar{\psi}(x) \boldsymbol{\gamma} \cdot \boldsymbol{D}_{\mathrm{lat}} \psi(x)-\frac{1}{2} r_{s} \zeta a^{5} \sum_{x} \bar{\psi}(x) \triangle_{\mathrm{lat}}^{(3)} \psi(x)
\end{aligned}
$$

We denote lattice fermions fields with $\psi$ to distinguish them from the continuum quark fields in Eqs. (1.1) and (1.3). The coupling $\zeta$ allows one to adjust $m_{2}=m_{1}$ in Eq. (1.1). The notation for $\boldsymbol{D}_{\text {lat }}, \triangle_{\text {lat }}^{(3)}$, etc., is as in Ref. [3]. The dimension-five interactions are [2, 3]

$$
\begin{aligned}
& S_{(5,0)}=S_{B}=-\frac{1}{2} c_{B} \zeta a^{5} \sum_{x} \bar{\psi}(x) i \boldsymbol{\Sigma} \cdot \boldsymbol{B}_{\mathrm{lat}} \psi(x), \\
& S_{(5,1)}=S_{E}=-\frac{1}{2} c_{E} \zeta a^{5} \sum_{x} \bar{\psi}(x) \boldsymbol{\alpha} \cdot \boldsymbol{E}_{\mathrm{lat}} \psi(x),
\end{aligned}
$$


where the notation $S_{B}$ and $S_{E}$ is from Ref. [3]. At dimension six and seven we introduce

$$
\begin{aligned}
& S_{(6,0)}=r_{E} a^{6} \sum_{x} \bar{\psi}(x)\left\{\boldsymbol{\gamma} \cdot \boldsymbol{D}_{\text {lat }}, \boldsymbol{\alpha} \cdot \boldsymbol{E}_{\text {lat }}\right\} \psi(x) \\
& +z_{E} a^{6} \sum_{x} \bar{\psi}(x) \gamma_{4}\left(\boldsymbol{D}_{\text {lat }} \cdot \boldsymbol{E}_{\text {lat }}-\boldsymbol{E}_{\text {lat }} \cdot \boldsymbol{D}_{\text {lat }}\right) \psi(x), \\
& S_{(6,1)}=c_{1} a^{6} \sum_{x} \bar{\psi}(x) \sum_{i} \gamma_{i} D_{i \mathrm{lat}} \triangle_{i \mathrm{lat}} \psi(x) \\
& +c_{2} a^{6} \sum_{x} \bar{\psi}(x)\left\{\boldsymbol{\gamma} \cdot \boldsymbol{D}_{\text {lat }}, \triangle_{\text {lat }}^{(3)}\right\} \psi(x) \\
& +c_{3} a^{6} \sum_{x} \bar{\psi}(x)\left\{\boldsymbol{\gamma} \cdot \boldsymbol{D}_{\text {lat }}, i \boldsymbol{\Sigma} \cdot \boldsymbol{B}_{\text {lat }}\right\} \psi(x) \\
& +z_{3} a^{6} \sum_{x} \bar{\psi}(x) \boldsymbol{\gamma} \cdot\left(\boldsymbol{D}_{\text {lat }} \times \boldsymbol{B}_{\text {lat }}+\boldsymbol{B}_{\text {lat }} \times \boldsymbol{D}_{\text {lat }}\right) \psi(x) \\
& +c_{E E} a^{6} \sum_{x} \bar{\psi}(x)\left\{\gamma_{4} D_{4 \mathrm{lat}}, \boldsymbol{\alpha} \cdot \boldsymbol{E}_{\text {lat }}\right\} \psi(x), \\
& S_{(7,0)}=c_{4} a^{7} \sum_{x} \bar{\psi}(x) \sum_{i} \triangle_{i \text { lat }}^{2} \psi(x) \\
& +c_{5} a^{7} \sum_{x} \bar{\psi}(x) \sum_{i} \sum_{j \neq i}\left\{i \Sigma_{i} B_{i \text { lat }}, \triangle_{j_{\text {lat }}}\right\} \psi(x) \\
& +r_{5} a^{7} \sum_{x} \bar{\psi}(x) \sum_{i} \sum_{j \neq i} i \Sigma_{i}\left[D_{j} B_{i} D_{j}\right]_{\mathrm{lat}} \psi(x) \\
& +z_{6} a^{7} \sum_{x} \bar{\psi}(x)\left(\triangle_{\text {lat }}^{(3)}\right)^{2} \psi(x) \\
& +z_{7} a^{7} \sum_{x} \bar{\psi}(x)\left\{\triangle_{\text {lat }}^{(3)}, i \boldsymbol{\Sigma} \cdot \boldsymbol{B}_{\text {lat }}\right\} \psi(x) \\
& +z_{7}^{\prime} a^{7} \sum_{x} \bar{\psi}(x)\left[D_{i} i \boldsymbol{\Sigma} \cdot \boldsymbol{B} D_{i}\right]_{\mathrm{lat}} \psi(x) \\
& +r_{7} a^{7} \sum_{x} \bar{\psi}(x) \boldsymbol{\gamma} \cdot \boldsymbol{D}_{\mathrm{lat}} i \boldsymbol{\Sigma} \cdot \boldsymbol{B}_{\mathrm{lat}} \boldsymbol{\gamma} \cdot \boldsymbol{D}_{\mathrm{lat}} \psi(x) \\
& +r_{7}^{\prime} a^{7} \sum_{x} \bar{\psi}(x)[\boldsymbol{D} \cdot(\boldsymbol{B} \times \boldsymbol{D})]_{\mathrm{lat}} \psi(x) \\
& +r_{B B} a^{7} \sum_{x} \bar{\psi}(x)\left(i \boldsymbol{\Sigma} \cdot \boldsymbol{B}_{\text {lat }}\right)^{2} \psi(x) \\
& +z_{B B} a^{7} \sum_{x} \bar{\psi}(x) \boldsymbol{B}_{\text {lat }} \cdot \boldsymbol{B}_{\text {lat }} \psi(x) \\
& -r_{E E} a^{7} \sum_{x} \bar{\psi}(x)\left(\boldsymbol{\alpha} \cdot \boldsymbol{E}_{\mathrm{lat}}\right)^{2} \psi(x) \\
& +z_{E E} a^{7} \sum_{x} \bar{\psi}(x) \boldsymbol{E}_{\mathrm{lat}} \cdot \boldsymbol{E}_{\mathrm{lat}} \psi(x) .
\end{aligned}
$$

In particular, in heavy-quark power counting the interactions in $S_{(7,0)}$ are commensurate with those in $S_{(6,1)}$. Most of these interactions have been discussed previously [5, 6], but the following is the first discussion of interactions with two chromomagnetic or chromoelectric fields, and the related interaction with coupling $c_{E E} .{ }^{1}$

By applying arbitrary field redefinitions one can make certain coefficients in the effective Lagrangian vanish, and their interactions are called redundant. A corresponding number of couplings

\footnotetext{
${ }^{1}$ At dimension six and higher one must also consider four-quark interactions, which we shall discuss elsewhere.
} 
in the lattice action may be chosen by convenience. For example, to obviate the doubling problem one sets $r_{s} \neq 0$ and $r_{t}=1$ in Eq. (3.2). One can show that all interactions with further time derivatives acting on quark and antiquark fields are redundant, so we omit them from Eqs. (3.3)(3.7). Among the rest there is some freedom on which ones to call redundant; with an eye towards efficiency in computing quark propagators we choose

$$
r_{E}=r_{5}=r_{7}=r_{7}^{\prime}=r_{B B}=r_{E E}=0,
$$

a choice that is substantiated further by the matching calculations discussed in the next section.

\section{Matching}

To find out how to adjust the other couplings, we must explicitly derive Eqs. (1.2) or, equivalently, Eqs. (1.4), and solve them for the couplings in the lattice action. Because the action includes interactions with two chromoelectric and two chromomagnetic fields, we have done so by working out the amplitude for Compton scattering. We find, not unexpectedly, that many couplings vanish at the tree level. To be specific, tree-level matching yields

$$
z_{E}=z_{3}=z_{6}=z_{7}=z_{7}^{\prime}=z_{B B}=z_{E E}=0,
$$

but these become non-trivial beyond the tree level.

The other couplings $-c_{B}, c_{E}, c_{E E}, c_{1}, c_{2}, c_{3}, c_{4}$, and $c_{5}$-must be non-zero, with an explicit, sometimes nontrivial, dependence on $\left(m_{0} a, \zeta, r_{s}\right)$. We do not have space to discuss the intermediate steps here, so we quote the final result of tree-level matching. We find

$$
\begin{aligned}
c_{B} & =r_{s} \\
c_{E} & =\frac{\zeta^{2}-1}{m_{0} a\left(2+m_{0} a\right)}+\frac{r_{s} \zeta}{1+m_{0} a}+\frac{r_{s}^{2} m_{0} a\left(2+m_{0} a\right)}{4\left(1+m_{0} a\right)^{2}} \\
c_{1} & =-\frac{1}{6} \zeta+c_{B} \frac{m_{0} a\left(2+m_{0} a\right)}{6\left(1+m_{0} a\right)} \\
c_{2}=c_{3} & =\frac{\zeta^{3}\left(\zeta^{2}-1\right)}{\left[2 m_{0} a\left(2+m_{0} a\right)\right]^{2}}-\frac{\zeta^{2}\left[\zeta+2 r_{s}\left(1+m_{0} a\right)-3 r_{s} \zeta^{2} /\left(1+m_{0} a\right)\right]}{8 m_{0} a\left(2+m_{0} a\right)} \\
& +\frac{3 r_{s}^{2} \zeta^{3}}{16\left(1+m_{0} a\right)^{2}}+\frac{m_{0} a\left(2+m_{0} a\right) r_{s}^{2} \zeta}{32\left(1+m_{0} a\right)^{2}}\left[\frac{r_{s} \zeta}{1+m_{0} a}-1\right] \\
c_{E E}\left[2+m_{0} a\left(2+m_{0} a\right)\right] & =\frac{\zeta\left(\zeta^{2}-1\right)\left(1+m_{0} a\right)}{\left[m_{0} a\left(2+m_{0} a\right)\right]^{2}}+\frac{c_{E} \zeta\left(\zeta^{2}-1\right)\left(1+m_{0} a\right)}{m_{0} a\left(2+m_{0} a\right)} \\
& +\frac{\zeta\left(r_{s} \zeta-1-m_{0} a\right)}{2 m_{0} a\left(2+m_{0} a\right)}+\frac{1}{2} r_{s} c_{E} \zeta^{2}-\frac{1}{4} c_{E}^{2} \zeta\left(1+m_{0} a\right) \\
c_{4} & =\frac{1}{24} r_{s} \zeta+\frac{1}{3} c_{B} \zeta \\
c_{5} & =\frac{1}{4} c_{B} \zeta
\end{aligned}
$$

Some aspects are not surprising: for example $c_{2}=c_{3}$, which makes sense because together they provide an interaction of the form $\bar{\psi}(\boldsymbol{\gamma} \cdot \boldsymbol{D})^{3} \psi$. Two out of the three couplings $\left(c_{4}, c_{5}, r_{5}\right)$ must be nonzero, essentially to correct errors from the $\boldsymbol{B}_{\text {lat }}$ in $S_{(5,0)}$. We choose $c_{4} \neq 0$ because we expect its interaction to be easier to compute than that with coupling $r_{5}$. 


\section{Outlook}

In several recent calculations with Fermilab quarks, the largest systematic uncertainty comes from a (conservative) estimate of heavy-quark discretization effects. With the improved action presented here, the same technique for estimating the uncertainties [9] suggests that these effects are reduced to a few percent [6]. To achieve this target, tree-level matching should suffice for $S_{(6,1)}$ and $S_{(7,0)}$. For $S_{(5,0)}, S_{(5,1)}$, and $S_{(6,0)}$ higher accuracy is needed, either at the one-loop [10,11] or (for $c_{B}$ in $S_{(5,0)}$ ) nonperturbative level [12].

\section{Acknowledgments}

M.B.O. is supported by Science Foundation Ireland grant 04/BRG/P0275. Fermilab is operated by Universities Research Association Inc., under contract with the U.S. Department of Energy.

\section{References}

[1] See, for example, A. S. Kronfeld, Uses of effective field theory in lattice QCD, in At the Frontier of Particle Physics: Handbook of QCD (World Scientific, Singapore, 2002), Vol. 4, edited by M. Shifman [hep-lat/0205021].

[2] B. Sheikholeslami and R. Wohlert, Improved continuum limit lattice action for QCD with Wilson fermions, Nucl. Phys. B 259 (1985) 572.

[3] A. X. El-Khadra, A. S. Kronfeld, and P. B. Mackenzie, Massive fermions in lattice gauge theory, Phys. Rev. D 55 (1997) 3933 [hep-lat/9604004].

[4] A. S. Kronfeld, Application of heavy-quark effective theory to lattice QCD I: Power corrections, Phys. Rev. D 62 (2000) 014505 [hep-lat/0002008].

[5] M. B. Oktay, A. X. El-Khadra, A. S. Kronfeld, P. B. Mackenzie, and J. N. Simone, A relativistic $O\left(a^{2}\right)$ improved action for heavy quarks, Nucl. Phys. Proc. Suppl. 119 (2003) 464 [hep-lat/0209150].

[6] M. B. Oktay, A. X. El-Khadra, A. S. Kronfeld, and P. B. Mackenzie, A more improved lattice action for heavy quarks, Nucl. Phys. Proc. Suppl. 129 (2004) 349 [hep-lat/0310016].

[7] N. H. Christ, M. Li, and H. W. Lin, Relativistic heavy quark effective action, hep-lat/0608006.

[8] G. P. Lepage, L. Magnea, C. Nakhleh, U. Magnea, and K. Hornbostel, Improved nonrelativistic QCD for heavy quark physics, Phys. Rev. D 46 (1992) 4052 [hep-lat/9205007].

[9] A. S. Kronfeld, Heavy quarks and lattice QCD, Nucl. Phys. Proc. Suppl. 129 (2004) 46 [hep-lat/0310063].

[10] M. A. Nobes and H. D. Trottier, One loop renormalization of Fermilab fermions, Nucl. Phys. Proc. Suppl. 119 (2003) 461 [hep-lat/0209017]; Progress in automated perturbation theory for heavy quark physics, Nucl. Phys. Proc. Suppl. 129 (2004) 355 [hep-lat/0309086].

[11] S. Aoki, Y. Kayaba, and Y. Kuramashi, A perturbative determination of mass-dependent $O(a)$ improvement coefficients in a relativistic heavy quark action, Nucl. Phys. B 697 (2004) 271 [hep-lat/0309161].

[12] H. W. Lin and N. Christ, Nonperturbatively determined relativistic heavy quark action, hep-lat/0608005. 